\title{
Traumatic brain injury is not associated with significant myocardial dysfunction: an observational pilot study
}

\author{
Karim Serri ${ }^{1,2^{*}}$, Malak El Rayes ${ }^{1}$, Geneviève Giraldeau ${ }^{3}$, David Williamson ${ }^{1,2}$ and Francis Bernard ${ }^{1,2}$
}

\begin{abstract}
Background: Myocardial dysfunction has been well described with catastrophic neurological events, such as subarachnoid hemorrhage and brain death. There is very limited data describing myocardial function in the context of traumatic brain injury (TBI), as no prospective study has yet examined this association. The objective of our study was to evaluate cardiac function using echocardiography in patients with clinically important TBI.

Methods: We conducted a prospective observational study of consecutive TBI patients admitted to the intensive care unit. All patients older than 16 years with moderate to severe TBI according to the Glascow Coma Scale (GCS) were eligible for the study. Only patients with a prior history of heart disease or cardiomyopathy or evidence of brain death on admission were excluded. A complete transthoracic echocardiogram was performed within 4 days of admission.
\end{abstract}

Results: Forty-nine patients (67\% males, median age 34 years) were included in the study. Forty-one patients had severe TBI (84 \%) with a median GCS of six, 44 patients (90\%) required mechanical ventilation and 36 (74\%) intracranial pressure monitoring. Hospital mortality was $18 \%$. No patients had global left ventricular dysfunction as defined by a left ventricular ejection fraction (LVEF) below $50 \%(95 \% \mathrm{Cl}, 0-0.07)$. Average LVEF was $65+/-4 \%$. Four patients (8\%) had regional wall motion abnormalities with preserved LVEF.

Discussion: The main finding of this study is the absence of clinically significant myocardial dysfunction in patients with moderate or severe TBI. Although myocardial dysfunction has been well described in a variety of neurological settings, it is possible that the young age of TBI patients and the absence of cardiovascular risk factors are protective against significant myocardial injury from catecholamine excess.

Conclusions: In a group of patients with clinically important TBI, we did not identify any significant cardiac dysfunction.

Keywords: Traumatic brain injury, Myocardial dysfunction, Echocardiography, Left ventricular ejection fraction

\section{Background}

Traumatic brain injury (TBI) is a major cause of death and disability in Western nations [1, 2]. Prognosis is dependent on damage that occurs at the time of the initial trauma, but also over the following days as secondary injury [3]. One potential contributing factor to secondary

\footnotetext{
* Correspondence: karim.serri@umontreal.ca

'Département de médecine, Hôpital du Sacré-Coeur de Montréal, Service de soins intensifs, Université de Montréal, 5400, boul Gouin ouest, Montréal H4J-1C5, Canada

${ }^{2}$ Centre de recherche Hôpital du Sacré-Coeur de Montréal, Université de Montréal, Montréal, Canada

Full list of author information is available at the end of the article
}

injury is autonomic dysfunction [4]. This phenomenon is thought to result from excessive adrenergic activity which most often causes abnormalities in heart rate, blood pressure and thermoregulation. In its most severe form, this excessive adrenergic activity can lead to myocardial dysfunction, which has been well characterized in the context of spontaneous subarachnoid hemorrhage (SAH). In this setting, cardiac manifestations ranging from asymptomatic electrocardiographic abnormalities to severe myocardial dysfunction [5-7] have been described and are believed to be related to catecholamine toxicity [8]. Myocardial dysfunction has also been described with other catastrophic 
neurological events, such as brain death in up to $40 \%$ of patients [9-11].

There is very limited data describing myocardial function in the context of TBI, as no prospective study has yet examined this association. Based on the literature on SAH and brain death, one would expect a relatively high incidence of myocardial injury. One recent study lends support to that hypothesis with a reported incidence of $22 \%$ of cardiac dysfunction in elderly patients with isolated TBI [12]. These findings are, however, limited by their retrospective nature and the older age of the studied population, not necessarily representative of young TBI patients.

Myocardial dysfunction in the setting of TBI could have an important impact as it may contribute to secondary injury by altering cerebral blood flow. Accordingly, the objective of our study was to evaluate cardiac function in patients with clinically important TBI. We hypothesized that cardiac dysfunction occurs frequently after TBI and that it is associated with the severity of the TBI.

\section{Methods \\ Setting}

We conducted a prospective observational study of consecutive TBI patients admitted to the intensive care unit (ICU) at Hôpital du Sacré-Coeur de Montréal, a 500-bed general hospital in Montreal, Canada. The institution is an academic level 1 trauma center affiliated with the University of Montreal, with more than 1600 trauma admissions per year and between 150 and 180 moderate or severe TBIs per year. The unit is an intensivist-run unit with 38 ICU beds, among which 10 are dedicated for neurological ICU patients. The study was approved by the local institutional review board (CER, Hôpital SacréCoeur de Montréal). Informed consent was obtained from the patients' next-of-kin.

\section{Study population}

All patients older than 16 years with moderate to severe TBI according to the Glascow Coma Scale (GCS) were eligible for the study. TBI was categorized as moderate (GCS 9-12) and severe (GCS 3-8) according to Brain Trauma Foundation guidelines. Only patients with a GCS of less than 13 were included in the study. Patients with a prior history of heart disease or cardiomyopathy and patients with evidence of brain death on admission were excluded. Patients whose ultrasound images were deemed uninterpretable were also excluded from the study.

\section{Patient care}

Patients were treated according to local institutional practices consistent with the Brain Trauma Foundation guidelines [13-15]. Intracranial pressure (ICP) monitoring was performed in patients in whom it was clinically indicated as per the treating neurosurgeon and intensivist. In patients with ICP monitoring, cerebral perfusion pressure was kept above $60 \mathrm{~mm} \mathrm{Hg}$ while maintaining ICP below $20 \mathrm{~mm} \mathrm{Hg}$. Mean arterial pressure was measured with an arterial line calibrated at the level of the tragus. $\mathrm{PaCO} 2$ was kept between $4.7-5.3 \mathrm{kPa}(35-40 \mathrm{~mm} \mathrm{Hg})$ in mechanically ventilated patients, and $\mathrm{O} 2$ saturation $>94 \%$. Efforts were made to keep patients euvolemic, to maintain natremia and glycemia normal and to prevent hyperthermia. Analgesia and sedation were used at the minimal required doses to allow optimal ICP control. Cerebral computed tomography $(\mathrm{CT})$ scans were performed on admission and as needed thereafter.

\section{Outcome measures}

Demographic, clinical, laboratory and electrocardiographic data were collected at the time of admission. Standard twelve lead electrocardiograms (ECG) were recorded daily for the first 4 days following admission. Troponin I levels were drawn daily for the first 4 days after admission with a level greater than $0.1 \mathrm{mcg} / \mathrm{l}$ considered abnormal. CK, CK-MB levels were measured as well. TBI severity was graded clinically according to the GCS after resuscitation and radiographically according to the Marshall [16] and Rotterdam scores [17]. Measurement of ICP was documented when available. An elevated ICP was defined as a non-stimulated spontaneous increase greater than $20 \mathrm{~mm}$ $\mathrm{Hg}$ for more than $5 \mathrm{~min}$.

A complete transthoracic echocardiogram was performed within the first days of admission, with assessment of left ventricular (LV) function and dimensions according to current guidelines [18]. The images were acquired in 5 standard views (parasternal long and short axis, apical 2-, 3- and 4-chamber) and stored digitally in cineloop format using a commercially available ultrasound system. LV ejection fraction was assessed visually and measured by the Simpson method when possible. LV diastolic function was assessed as recommended by the American Society of Echocardiography by calculating E/A and E/e' ratios [18]. Briefly, using pulsed-wave Doppler, the early mitral filling wave (E) and the atrial contraction wave (A) were measured and the E/A ratio was calculated. Using tissue Doppler, the early mitral filling wave (e') was measured at the lateral mitral annulus and the E/e' ratio was calculated. Right ventricular function was evaluated by measuring tricuspid annular velocity (S') by tissue Doppler [19]. LV dimensions, left atrial dimensions and the presence of valvular disease were assessed according to recommendations. Two independent blinded experienced observers unaware of patient clinical condition reviewed all echocardiograms.

The main study outcome was the presence of LV dysfunction as defined by a $\mathrm{LVEF}<50 \%$. As secondary outcomes, we measured troponin levels and studied ECG patterns to determine if there was an association with LV dysfunction. 


\section{Statistical analysis}

Based on data from SAH patients and brain death patients, we estimated the prevalence of myocardial dysfunction to be approximately $20 \%$. We planned to enroll 50 patients in this pilot study, which would have allowed to detect myocardial dysfunction with a precision of $11 \%$ with a confidence interval of $95 \%$. Continuous data is presented as mean \pm SD or median (IQR) according to distribution and categorical data is presented as proportions.

\section{Results}

\section{Baseline characteristics}

Between October 2009 and January 2012, a total of 64 patients were screened and 49 patients were included in the study. Exclusions were mainly due to poor ultrasound image quality. Baseline characteristics are presented in Table 1 . The majority of patients were men $(67 \%)$ with a median age of 34 years (IQR 27.5, range 16-81). Four patients were older than 65 years, and 6 were older than 50. The mechanism of TBI was most frequently a motor vehicle accident, accounting for $58 \%$ of the cases, with the remainder being due to falls, fights, and falling objects. Forty-one patients had severe TBI (84 \%). The mean GCS was $6+/-3$ (range 3-13). Thirty-six patients (74\%) required ICP monitoring, and 12 had an elevated ICP (25\%). Twenty-four patients (49\%) had SAH and 20 (41 \%) had a subdural hematoma. Thirteen patients (27\%) required a craniectomy. Associated traumatic injuries were the following: 12 patients with orthopedic fractures, 12 with maxillofacial fractures, 7 with spinal fractures, and 13 with other injuries (the vast majority representing pulmonary contusions). Thirty-seven patients (76\%) received vasopressors (none received inotropes) and 44 (90\%) were

Table 1 Baseline characteristics

\begin{tabular}{ll}
\hline Median age (range) & $34(16-81)$ \\
Male (\%) & 67 \\
Mean GCS & $6(+/-3)$ \\
Severe TBI (\%) & 84 \\
ISS & $32+/-11$ \\
Subarachnoid hemorrhage (\%) & 49 \\
Subdural hematoma (\%) & 41 \\
Mean Marshall score & $2.8+/-1.5$ \\
Mean Rotterdam score & $3.5+/-1.2$ \\
ICP monitoring (\%) & 74 \\
Craniectomy & 27 \\
Vasopressors (\%) & 76 \\
Mechanical ventilation (\%) & 90 \\
Mortality (\%) & 18 \\
\hline
\end{tabular}

GCS Glasgow Coma Scale, TBI Traumatic brain injury, ISS Injury Severity Score, ICP Intracranial Pressure mechanically ventilated. There were 9 deaths during the index hospital admission (18 \%).

\section{Echocardiographic results}

Echocardiography was performed at a median of $49 \mathrm{~h}$ (8-189) post trauma, and 18 patients had their echochardiograms within $48 \mathrm{~h}$. Table 2 shows the results of the echocardiographic findings. LV systolic and diastolic dimensions were normal. The average LVEF was $65+/-$ $4 \%(n=49)$. No patients had global LV dysfunction as defined by a LVEF below $50 \%$ (95\% CI, 0-0.07). Four patients $(8 \%)$ had regional wall motion abnormalities with preserved LVEF. Diastolic function was normal in 39 patients, could not be determined in eight patients, and two patients had mild diastolic dysfunction. The estimated left ventricular filling pressures were normal with an average lateral E/e' of $5.7+/-1.5(n=39)$ (normal $\left.\mathrm{E} / \mathrm{e}^{\prime}<8\right)$. No patient had right ventricular dysfunction. Five patients were found to have mild valvular regurgitation, no patient had significant valvular abnormalities.

\section{ECG and troponin}

Troponin I levels and CK-MB levels were available in 43 and 40 patients respectively. Fifteen patients (31\%) had an elevated troponin level. The median maximum troponin I concentration was $0.05 \mathrm{mcg} / \mathrm{l}$ (IQR 0.22, range $0.01-$ 22). Maximal troponin elevation occurred at an average of 1.9 days. The median maximal CK-MB was $15 \mathrm{U} / \mathrm{l}$ (IQR 21.7, range 2.8-212.4). Of the four patients with regional wall motion abnormalities, none of them had elevated troponin levels, and none of these patients died. As for cardiac risk factors, three patients had hypertension, one was a known diabetic, and eight were smokers.

ECG abnormalities were present in 28 patients (57\%), for the most part non specific ST segment or T waves changes. Five patients had ECG changes suggesting acute ischemia, none of which had enzymatic criteria for myocardial infarction or required coronary angiography. QT interval was prolonged in $22.4 \%$ of patients, with

Table 2 Echocardiographic findings

\begin{tabular}{|c|c|c|}
\hline & Study patients & Reference range \\
\hline LVEF (\%) & $65(+/-4)$ & $\geq 55$ \\
\hline LVEDD (mm) & $46(+/-6)$ & F 38-52, M 42-58 \\
\hline $\operatorname{LVESD}(\mathrm{mm})$ & $30(+/-6)$ & F 22-35, M 25-40 \\
\hline$E / A$ & $1.5(+/-0.5)$ & $0.8-1.5$ \\
\hline Lateral E/e' & $5.7(+/-1.5)$ & $\leq 8$ \\
\hline Right ventricular $\mathrm{S}^{\prime}(\mathrm{cm} / \mathrm{s})$ & $15(+/-3)$ & $\geq 10$ \\
\hline
\end{tabular}


severe prolongation (QTc $>500 \mathrm{~ms})$ in $8 \%$. Average QTc was $456+/-43 \mathrm{~ms}$.

\section{Discussion}

The main finding of this study is the absence of clinically significant myocardial dysfunction in patients with moderate or severe TBI. Although troponin elevation and ECG abnormalities are frequent, they do not appear to be associated with myocardial dysfunction. This is to our knowledge the first prospective study of myocardial dysfunction and TBI.

Myocardial dysfunction has been well described in a variety of neurological settings. Aneurysmal SAH has been the most studied example with impaired systolic function reported in 8-53\% of patients. In an echocardiographic study of 300 patients with $\mathrm{SAH}$, Kothavale et al. described wall motion abnormalities in $18 \%$ of patients and in $35 \%$ of patients with high-grade $\mathrm{SAH}$ [5]. A reduced LVEF $(<50 \%)$ was found in $8 \%$ of patients. In a study of 294 patients with SAH, Urbaniak et al found impaired systolic function (LVEF $<40 \%$ ) in $53 \%$ of patients [6]. The mechanism responsible for myocardial injury in this setting is thought to be catecholamine toxicity $[8,20,21]$. The sympathetic discharge associated with SAH causes a specific form of myocyte injury called contraction band necrosis [22]. These findings have been noted both in experimental and human studies [23-25]. Similar pathophysiologic mechanisms may be involved in stress cardiomyopathy and brain death-associated myocardial dysfunction $[26,27]$.

Though well described in these settings, there is very limited data in patients with TBI, as only two retrospective studies have evaluated the association between TBI and cardiac function. In a preliminary retrospective study of 51 patients with severe brain injury in whom transesophageal echocardiography was performed, LV dysfunction was observed in $14 \%$ of patients, and severe LV dysfunction in $8 \%$ [10]. Another $16 \%$ of patients were found to have regional wall motion abnormalities with preserved global LV function. Of note in this study, $45 \%$ of patients had non traumatic brain injury and all patients eventually evolved towards brain death. Although brain death diagnosis was not yet made at the time of the echocardiogram, it is possible that some patients may already have been brain dead. Furthermore, because of the retrospective nature of the study, it was not possible to determine if patients had pre-existing heart disease. A recent retrospective study of 139 patients with TBI reported abnormal echocardiograms in $22 \%$ of patients and reduced LVEF in $12 \%$ of cases [12]. There are however significant differences with the present study: patients were older (58 years on average) and the predominant traumatic lesions were subdural hematomas (63\%). Older age and greater prevalence of cardiovascular risk factors could explain some of the echocardiographic abnormalities. Furthermore, the fact that echocardiograms were not part of a protocol but ordered on clinical grounds, and the retrospective nature of the study could have introduced significant selection bias. For these reasons, cardiac dysfunction may have been overestimated.

We did not observe any significant myocardial dysfunction in our patient population. All parameters of cardiac function, including systolic and diastolic function, left and right ventricular function, were normal. Diastolic function is often an early marker of myocardial dysfunction appearing before any systolic abnormalities. Taken together, these findings are consistent overall with the absence of any clinically significant cardiac dysfunction following TBI. Although no prospective human data is available, a recent animal study did not identify any functional or histopathological abnormalities in a rat model of TBI [28]. Troponin elevation however was frequent in our patients, but was not associated with TBI severity or myocardial dysfunction. In a retrospective review of 420 patients with TBI, Salim et al. found increased troponin I levels in $29.8 \%$ of patients using a cut-off of $0.3 \mathrm{ng} / \mathrm{ml}$, very similar to our findings. The authors describe an association between troponin levels and severity of brain injury as well as outcome. Interestingly, beta-blocker use was found to be protective [29]. We observed an elevated proportion of ECG abnormalities in our patients, for the most part non specific changes and QT prolongation. These findings are similar to what has been described in SAH [30].

The reason why other forms of brain injury are associated with cardiac dysfunction and not TBI is unclear. As mentioned above, brain-heart interactions have been well documented in SAH. Severity of injury does not appear to play a role, as our study mostly involved patients with severe TBI. Considering the pathophysiology of myocardial dysfunction in SAH, one could postulate that TBI be associated with lower levels of catecholamines, which again appears unlikely considering the severity of disease. However, it is possible that the younger age of TBI patients (and the associated absence of cardiovascular risk factors) compared to typical SAH patients is protective against significant myocardial injury from catecholamine excess.

\section{Limitations}

The main limitations of our study is the small sample size, which may have limited the power to detect an association between TBI and significant cardiac dysfunction. However, none of the echocardiographic parameters were in favour of myocardial dysfunction. Studies were performed $49 \mathrm{~h}$ post trauma, which may have been late to identify very early myocardial dysfunction. Based on data on SAH 
patients, one would expect cardiac dysfunction to persist at least several days [31]. Hence, if myocardial dysfunction occurs very early in TBI, it does not appear to be clinically significant.

\section{Conclusion}

Although troponin elevation and ECG abnormalities are frequent in patients with moderate to severe TBI, we did not identify any significant cardiac dysfunction. It is possible that the young age of patients with TBI and the absence of cardiovascular risk factors are protective against significant myocardial injury from catecholamine excess. Further studies to confirm these findings and to understand the underlying pathophysiology are needed.

\section{Abbreviations}

ECG: electrocardiograms; GCS: Glascow Coma Scale; ICP: Intracranial pressure; ICU: intensive care unit; LV: left ventricular; LVEF: left ventricular ejection fraction; SAH: subarachnoid hemorrhage; TBI: traumatic brain injury.

\section{Competing interests}

The authors declare that they have no competing interests.

\section{Authors' contributions}

MER performed data analysis and echocardiographic image analysis, and helped draft the manuscript. GG performed data analysis and echocardiographic image analysis. FB reviewed radiologic data and helped draft the manuscript. DW performed statistical analysis. KS conceived the study, participated in its design and coordination, and drafted the manuscript. All authors read and approved the final manuscript.

\section{Acknowledgments}

The authors would like to thank the staff of the echocardiography laboratory at Hôpital du Sacré-Coeur de Montréal, as well as the ICU staff for their implication in this project.

\section{Author details}

'Département de médecine, Hôpital du Sacré-Coeur de Montréal, Service de soins intensifs, Université de Montréal, 5400, boul Gouin ouest, Montréal H4J-1C5, Canada. ${ }^{2}$ Centre de recherche Hôpital du Sacré-Coeur de Montréal, Université de Montréal, Montréal, Canada. ${ }^{3}$ Institut de Cardiologie de Montréal, Université de Montréal, Montréal, Canada.

\section{Received: 30 July 2015 Accepted: 27 February 2016}

Published online: 16 March 2016

\section{References}

1. Marik PE, Varon J, Trask T. Management of head trauma. Chest. 2002; 122(2):699-711

2. Coronado VG et al. Surveillance for traumatic brain injury-related deathsUnited States, 1997-2007. MMWR Surveill Summ. 2011;60(5):1-32.

3. Maas Al, Stocchetti N, Bullock R. Moderate and severe traumatic brain injury in adults. Lancet Neurol. 2008;7(8):728-41.

4. Hinson HE, Sheth KN. Manifestations of the hyperadrenergic state after acute brain injury. Curr Opin Crit Care. 2012;18(2):139-45.

5. Kothavale $A$ et al. Predictors of left ventricular regional wall motion abnormalities after subarachnoid hemorrhage. Neurocrit Care. 2006;4(3): 199-205.

6. Urbaniak K et al. Cardiac complications after aneurysmal subarachnoid hemorrhage. Surg Neurol. 2007;67(1):21-8. discussion 28-9.

7. Naidech AM et al. Cardiac troponin elevation, cardiovascular morbidity, and outcome after subarachnoid hemorrhage. Circulation. 2005;112(18):2851-6.

8. Banki NM et al. Acute neurocardiogenic injury after subarachnoid hemorrhage. Circulation. 2005:112(21):3314-9.

9. Dujardin KS et al. Myocardial dysfunction associated with brain death: clinical, echocardiographic, and pathologic features. J Heart Lung Transplant. 2001;20(3):350-7.
10. Huttemann E et al. Left ventricular dysfunction in lethal severe brain injury: impact of transesophageal echocardiography on patient management. Intensive Care Med. 2002;28(8):1084-8.

11. Borbely XI, et al. Temporal changes in left ventricular systolic function and use of echocardiography in adult heart donors. Neurocrit Care. 2015;23(1): 66-71. doi:10.1007/s12028-014-0101-x

12. Prathep $\mathrm{S}$ et al. Preliminary report on cardiac dysfunction after isolated traumatic brain injury. Crit Care Med. 2014;42(1):142-7.

13. Brain Trauma F et al. Guidelines for the management of severe traumatic brain injury. I. Blood pressure and oxygenation. J Neurotrauma. 2007;24 Suppl 1:S7-13.

14. Brain Trauma F et al. Guidelines for the management of severe traumatic brain injury. VIII. Intracranial pressure thresholds. J Neurotrauma. 2007;24 Suppl 1:S55-8.

15. Brain Trauma F et al. Guidelines for the management of severe traumatic brain injury. IX. Cerebral perfusion thresholds. J Neurotrauma. 2007:24 Suppl 1:S59-64.

16. Marshall LF et al. The diagnosis of head injury requires a classification based on computed axial tomography. J Neurotrauma. 1992;9 Suppl 1 S287-92.

17. Maas Al et al. Prediction of outcome in traumatic brain injury with computed tomographic characteristics: a comparison between the computed tomographic classification and combinations of computed tomographic predictors. Neurosurgery. 2005;57(6):1173-82. discussion 1173-82.

18. Lang RM et al. Recommendations for chamber quantification: a report from the American Society of Echocardiography's Guidelines and Standards Committee and the Chamber Quantification Writing Group, developed in conjunction with the European Association of Echocardiography, a branch of the European Society of Cardiology. J Am Soc Echocardiogr. 2005;18(12): 1440-63.

19. Rudski LG et al. Guidelines for the echocardiographic assessment of the right heart in adults: a report from the American Society of Echocardiography endorsed by the European Association of Echocardiography, a registered branch of the European Society of Cardiology, and the Canadian Society of Echocardiography. J Am Soc Echocardiogr. 2010;23(7):685-713. quiz 786-8.

20. Lee $\mathrm{VH}$ et al. Mechanisms in neurogenic stress cardiomyopathy after aneurysmal subarachnoid hemorrhage. Neurocrit Care. 2006;5(3):243-9.

21. Tung $P$ et al. Predictors of neurocardiogenic injury after subarachnoid hemorrhage. Stroke. 2004;35(2):548-51.

22. Karch SB, Billingham ME. Myocardial contraction bands revisited. Hum Pathol. 1986:17(1):9-13.

23. Hawkins WE, Clower BR. Myocardial damage after head trauma and simulated intracranial haemorrhage in mice: the role of the autonomic nervous system. Cardiovasc Res. 1971;5(4):524-9.

24. Jacob WA, Van Bogaert A, De Groodt-Lasseel MH. Myocardial ultrastructure and haemodynamic reactions during experimental subarachnoid haemorrhage. J Mol Cell Cardiol. 1972:4(4):287-98.

25. Doshi R, Neil-Dwyer G. Hypothalamic and myocardial lesions after subarachnoid haemorrhage. J Neurol Neurosurg Psychiatry. 1977:40(8):821-6.

26. Wittstein IS et al. Neurohumoral features of myocardial stunning due to sudden emotional stress. N Engl J Med. 2005;352(6):539-48.

27. Novitzky $D$ et al. Prevention of myocardial injury during brain death by total cardiac sympathectomy in the Chacma baboon. Ann Thorac Surg. 1986; 41(5):520-4.

28. Najafipour $\mathrm{H}$ et al. Traumatic brain injury has not prominent effects on cardiopulmonary indices of rat after 24 hours: hemodynamic, histopathology, and biochemical evidence. Iran Biomed J. 2014;18(4):225-31.

29. Salim A et al. Significance of troponin elevation after severe traumatic brain injury. J Trauma. 2008;64(1):46-52.

30. Mayer SA et al. Electrocardiographic markers of abnormal left ventricular wall motion in acute subarachnoid hemorrhage. J Neurosurg. 1995;83(5): 889-96.

31. Salem $\mathrm{R}$ et al. Subarachnoid hemorrhage induces an early and reversible cardiac injury associated with catecholamine release: one-week follow-up study. Crit Care. 2014;18(5):558. 